\title{
ASPECTS REGARDING THE BEHAVIOR OF TOMATOES GRAFTED ON POTATOES
}

\author{
Daniela Giosanu $^{1^{*}}$, Florina Uleanu ${ }^{1^{*}}$, Ştefania Trăneci ${ }^{1}$, Mădălina Vulpe ${ }^{1}$ \\ ${ }^{1}$ University of Pitesti, Târgul din Vale Street, no 1, Piteşti Romania
}

Current Trends in

Natural Sciences

\begin{abstract}
Over time, different ways have been used to increase vegetable production per unit area; from the crop systems used (field crops, protected crops and forced crops), to intensive land use (successive, associated, double and intercropping) and to the technologies applied to them. In this paper we aimed to observe some aspects related to the behavior of tomatoes grafted on potatoes.The biological material was represented by: 'Sweet Million F1' cherry tomato hybrid variety, "Carrera" white potatoes, "Memphis" red potatoes, "Blue Star" hybrid purple potatoes. The experimental variants were: tomato control variant, potatoes control variants and tomato variant grafted on red, white and purple potatoes. It was observed that the best grafting was the tomato version grafted on the red potato. The control potato variants had the highest production compared to the grafted ones.The control tomato variant registered the highest number of flowers and formed the most fruits. The three grafted variants had a lower degree of fruit binding compared to the control variant.
\end{abstract}

Keywords: grafting, potato, tomato

\section{INTRODUCTION}

The needs of consumption and daily use of vegetables, by people in diets and not only, especially raw, fresh or processed, have contributed to the production of many cropping systems. These cropping systems have the role of staggering production over as long a period as possible during the year.

Solanaceae vegetable species can multiply by several methods; these methods can be grouped into classical or conventional methods (direct sowing, through seedlings, planted tubers) and unconventional methods (grafting). The advantages that this last method gives them are: it expands the cultivation area of valuable varieties, it diversifies the quality of the fruits, the productivity, the longevity, the precocity and the vigor (Arefin et al., 2019).

The vegetable grafting in our country is quite rare, but this method is used to reduce infections caused by pathogens, increase drought resistance, increase the absorption of nutrients from the soil and more recently to obtain two vegetable products at the same time. and on the same surface. This method is used for vegetable plants grown mainly in protected areas (greenhouses and solariums) (Reyes-Cabrera et al, 2018). 
From an agronomic point of view, grafting is important because it can combine the surface characteristics (fruit size) with the underground characteristics (root system) (Petran and Hoover, 2013). Due to the use of vigorous rootstock genotypes, the root system is strong and the absorption of water and minerals increases compared to grafted plants (Zeist et al., 2017).

The ecological requirements necessary for a good growth and development of tomatoes are air and soil temperature, intensity and duration of light period, air humidity (Helyes et al., 2012). Tomatoes are thermophilic, with different requirements depending on the phenophase. The two "sensitive" phases: the induction of the first inflorescence on the stem (first phase) and the number of flowers within it (second phase) are of particular importance. The lack of light during growth, development and fruit ripening leads to elongation of plants, flower anomalies and a delay in fruit ripening.

In the case of potatoes, heat is the main factor on which production depends, the optimal level for the development and growth of tubers is $15-18{ }^{\circ} \mathrm{C}$. The tuberization process takes place in conditions of intense lighting and corresponds phenologically with the budding-flowering phase.

The aim of this study was to observe the advantages of the split grafting method, using the "Sweet Million F1" cherry tomato variety as graft and the three types of potatoes (white, red and purple) as rootstocks.

\section{MATERIALS AND METHODS}

The experimental research activity took place in a solarium belonging to Salpitflor Green S.A., Pitesti, Arges. The solarium is equipped with an air recirculation system, sensors for the upper opening of the hatch along its entire length, a manual system for side opening, a control panel for observing temperature, humidity, speed and wind direction.

The biological material was represented by: "Sweet Million F1" cherry tomato hybrid variety, "Carrera" white potatoes, "Memphis" red potatoes, "Blue Star" hybrid purple potatoes.

The cherry tomato variety "Sweet Million F1" is a hybrid variety, with undetermined growth, suitable for early productions in greenhouses and solariums, but also in the open field. The variety is very productive, with a very high ability to bind fruits, forming double and triple bonds. The fruits are red, round, smooth, weigh between 10-30 grams, are resistant to transport and storage. The plant is resistant to the Verticilium tomato mosaic virus.

The white potatoes "Carrera" variety has the following characteristics: early maturity, oval shape, the color of the skin and the flesh light yellow, the size of the tubers is medium to very large, can form between 9-11 tubers.

"Memphis" variety potatoes show semi-early maturity, have an oval to long shape, red peel color, yellow pulp color, tuber size is medium to very large and can form between 9-11 tubers.

"Blue Star" hybrid purple potatoes have a semi-early maturity, oval to long shape, dark purple peel color, purple pulp color, tuber size are medium and can form between 12-14 tubers

The experimental variants were: tomato control variant (V.M.T), white potato control variant (V.M.C.A), red potato control variant (V.M.C.R), purple potato control variant (V.M.C.M), tomato variant grafted on white potato (V.T.C.A), tomato variant grafted on red potato (V.T.C.R) and tomato variant grafted on purple potato (V.T.C.M).

Each experimental variant had 5 repetitions; the geographical and climatic conditions were the same for all, as was the technology applied. 


\section{RESULTS AND DISCUSSIONS}

The study began by observing the grafting data and clamping by grafting data, for each repetition of each variant. The best clamping by grafting was remark at the tomato version grafted on the red potato, followed by the tomato version grafted on the purple potato; the weakest graft was the tomato version grafted on the white potato.

The average weight of tubers at harvest compared to the average weight at planting recorded significant increases and decreases. The control potato variants had the highest production compared to the grafted ones: the red potato control variant registered the highest production, followed by the purple potato control variant and the lowest production being the white potato control variant (see figure 1).

Among the grafted potato variants, the grafted variant of red potato had the highest production, followed by the grafted variant of white potato, and the grafted variant of purple potato recorded the lowest production of all variants (being even lower than at planting).

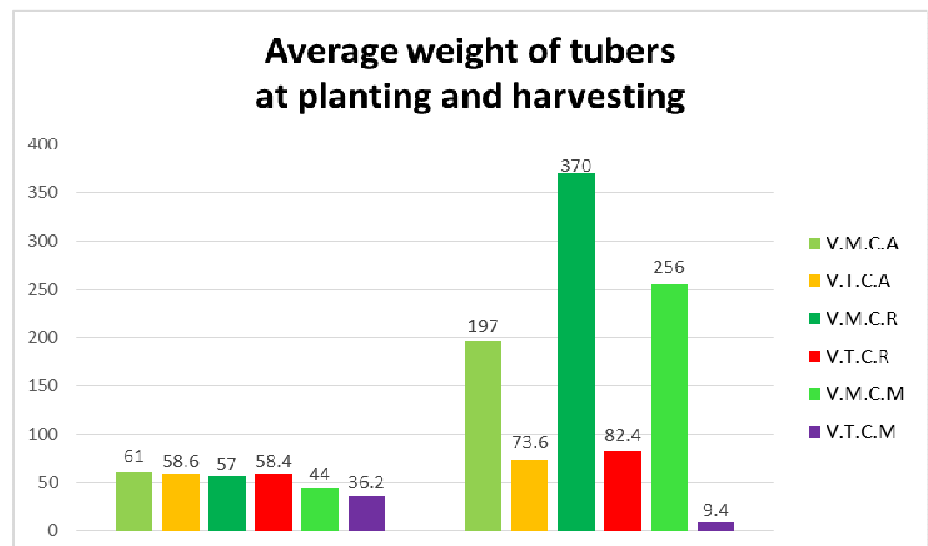

Figure 1. The average weight of tubers (at planting and harvest)

The control tomato variant registered the highest number of flowers, followed by the variant grafted on red potato, and the variant grafted on white potato presented the lowest number (figure 2).

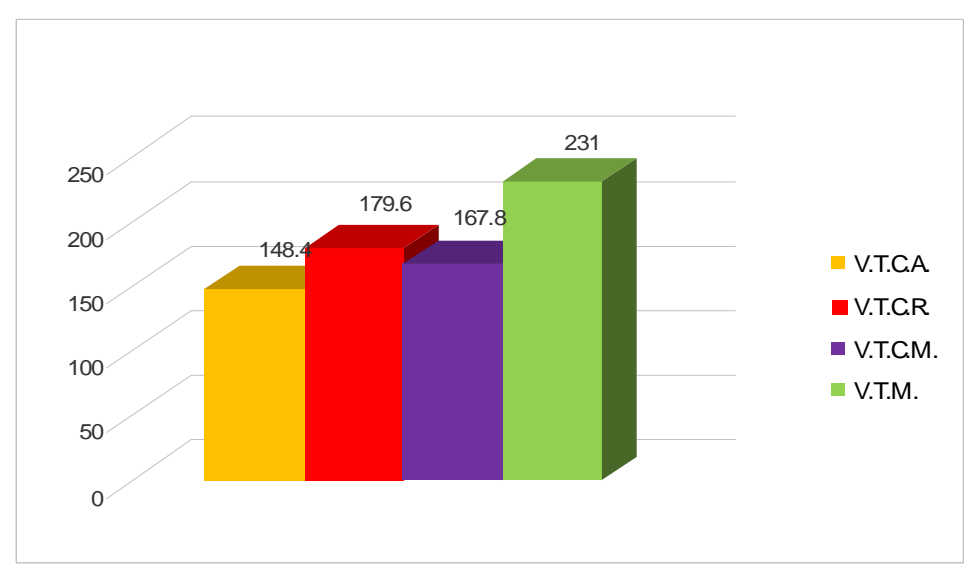

Figure 2. The average number of flower

The control tomato variant formed the most fruits, followed by the graft variant on red potato, while the tomato variant grafted on white potato formed the least (figure 3). 


\section{Current Trends in Natural Sciences}

Vol. 9, Issue 17, pp. 205-209, 2020

https://doi.org/10.47068/ctns.2020.v9i17.025

Current Trends in Natural Sciences (on-line)

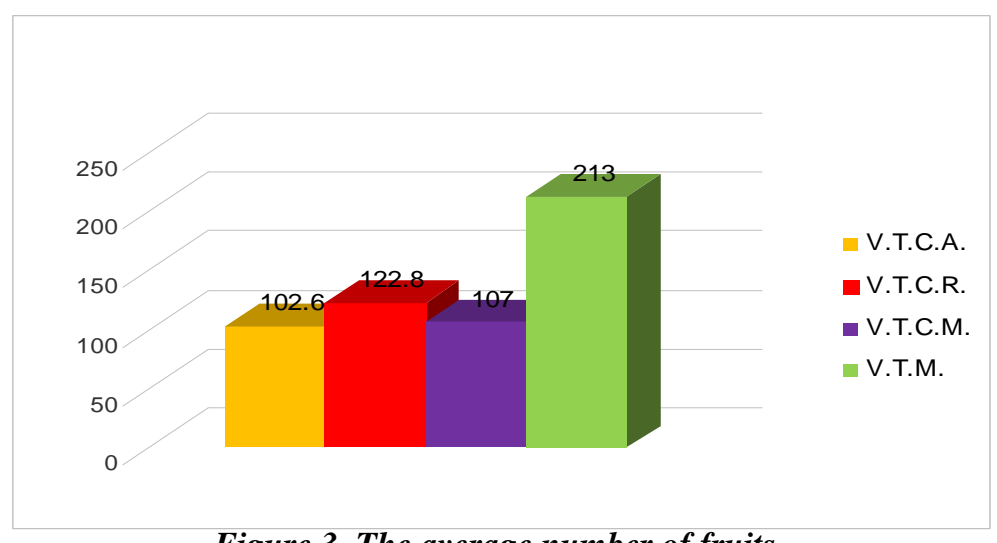

Figure 3. The average number of fruits

The three grafted variants had a lower degree of fruit binding compared to the control variant. The highest degree of binding of the fruits, from the grafted variants, had the variant grafted on white potato, for the other values being close (figure 4).

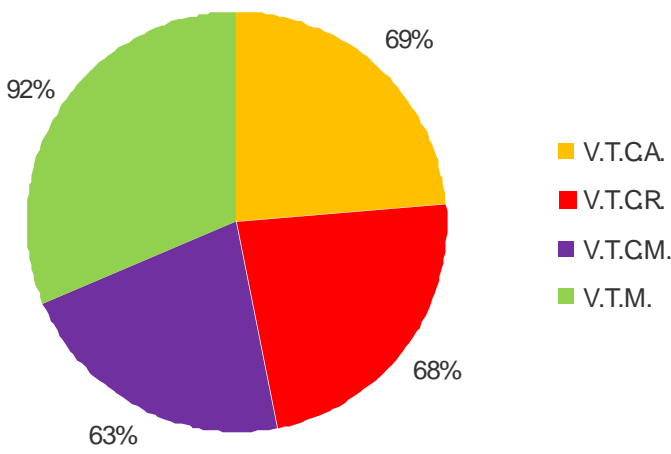

Figure 4. The degree of fruit binding / variant

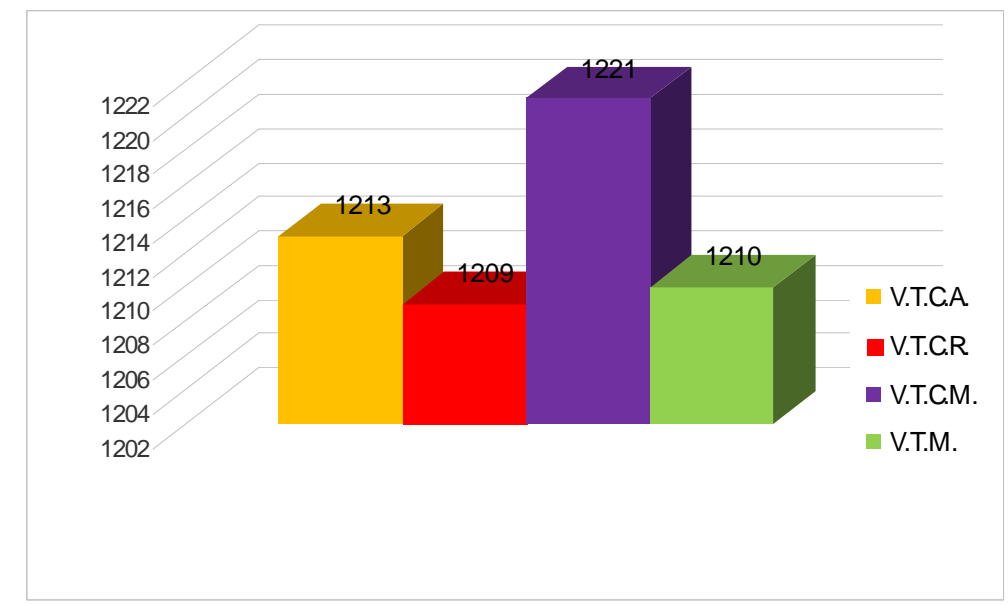

Figure 5. The average weight of 100 fruits tomato grafted on potatoes 
The largest harvested production, among the grafted variants, was held by the tomato variant grafted on purple potato, followed by the tomato variant grafted on red potato (figure 5). The fruits of the control tomato variant and the tomato variant grafted on red potato had an approximately equal weight.

\section{CONCLUSIONS}

The best results for grafting were red potato varieties.

Most flowers and fruits, among the grafted variants, were recorded on tomatoes grafted on red potatoes.

The tomato grafted on purple potato variant had the lowest degree of fruit binding.

The average weight of tomato fruit on each variant was largely influenced by the amount of water in the fruit.

The three grafted variants had a lower degree of fruit binding compared to the control variant. The tomato grafted on red potato variant is recommended for a high production of fruits and tubers.

\section{REFERENCES}

Arefin, S. M. Anamul Zeba, Naheed Solaiman, Abul Naznin, Tahera Azad, Obyedul Tabassum, Mourita Park, Cheol. (2019). Evaluation of Compatibility, Growth Characteristics, and Yield of Tomato Grafted on Potato ('Pomato'). Horticulturae. 5, 37. 10.3390/horticulturae5020037.

Helyes L, Lugasi A, Pék Z. (2012). Effect of irrigation on processing tomato yield and antioxidant components. Turkish Journal of Agriculture and Forestry 36, 702-709;

Petran, Andrew and Hoover, Emily. (2013). Solanum torvum as a Compatible Rootstock in Interspecific Tomato Grafting. Journal of Horticulture. 01. 10.4172/2376-0354.1000103.

Reyes-Cabrera, Antonio Robledo-Torres, Valentín Valdez-Aguilar, Luis De la Fuente, Marcelino Ramirez Godina, Francisca Sandoval Rangel, Alberto. (2018). Yield and quality of hybrid tomato grafted and cultivated under shade mesh and greenhouse. Ecosistemas y Recursos Agropecuarios. 5, 89-95. 10.19136/era.a5n13.1050.

Zeist, Andre Resende, Juliano Silva, Israel Zanin, Daniel Faria, Cacilda Giacobbo, Clevison. (2017). Survival and compatibility of tomato grafted on solanaceous plants and by different grafting methods. Científica. 45. $10.15361 / 1984-5529.2017 \mathrm{v} 45 \mathrm{n} 3 \mathrm{p} 278-283$. 